\title{
Absceso de Psoas Secundario a un Adenocarcinoma Mucinoso de Colon Perforado: Semiología.
}

\author{
Lina M. Ortiz ${ }^{1}$, Gonzalo B. Ferreira ${ }^{2}$, Andrés M. Duarte ${ }^{3}$ \\ ${ }^{1}$ Estudiante de Medicina. Undécimo semestre. Fundación Universitaria Juan N. Corpas. Bogotá, Colombia \\ ${ }^{2}$ Docente. MD. Especialista en Medicina Familiar. Fundación Universitaria Juan N. Corpas. Bogotá, Colombia \\ ${ }^{3}$ Médico Patólogo, Clínica Corpas. Docente Fundación Universitaria Juan N. Corpas. Bogotá, Colombia
}

\section{RESUMEN}

El absceso de psoas secundario a adenocarcinoma mucinoso de colon perforado, es una entidad poco frecuente que ocurre en el 1,8\% de la población, lo que dificulta su diagnóstico e incrementa su morbimortalidad (1).

A nuestra institución ingresa un paciente con diagnóstico de absceso de psoas de etiología desconocida pálido en regulares condiciones generales, antecedentes de drenajes a repetición, al examen físico presencia de catéter sobre cresta iliaca derecha con salida de escaso líquido seroso purulento, paraclínicos con evidencia de anemia normocítica heterogenia, trombocitosis y eosinofilia.

Como posible diagnostico se consideró espondilodiscitis, se realizó una radiografía de tórax para descartar tuberculosis pulmonar y RMN (Resonancia Magnética Nuclear) que reveló solución de continuidad en la pared posterior del ciego con colección que se extendía en la pared lateral de la pelvis. Afectando el componente iliaco del músculo psoas iliaco derecho, hallazgo sugestivo a lesión tumoral, probablemente adenocarcinoma mucinoso de ciego perforado.

Paciente se lleva a hemicolectomia derecha con reporte histopatológico de tumor en el ciego con perforación macroscópica presente de tipo adenocarcinoma de patrón mucinoso de alto grado con pobre respuesta linfoide, extensión hasta la serosa con perforación, metástasis a 2 ganglios e implantes tumorales al mesogastrio, clasificación pT4N1M1. Se instauro manejo con drenaje, antibioticoterapia, hierro y se inició proceso de remisión a clínica oncológica.

El caso nos permitió reconocer el rendimiento operativo de las pruebas diagnósticas y de la importancia de su integración con la semiología del paciente, elementos que fueron fundamentales para llegar a un diagnóstico certero.

Palabras clave: Absceso de psoas, adenocarcinoma mucinoso, supuración.

\section{ABSTRACT}

\section{Abscess of Psoas Secondary to a Mucinous Adenocarcinoma of Perforated Colon: Semiology. Case Report.}

Psoas abscess secondary to perforated colon mucinous adenocarcinoma is a rare entity that occurs in $1.8 \%$ of the population, which makes it difficult to diagnose and increases morbidity and mortality (9).

A patient with a diagnosis of psoas abscess of unknown pale etiology in regular general conditions, a history of recurrent drainage, a physical examination, presence of a catheter on the right iliac crest with leakage of scarce purulent serous fluid, paraclinics with evidence of anemia. normocytic heterogeneity, thrombocytosis and eosinophilia.

As a possible diagnosis, spondylodiscitis was considered, a chest $\mathrm{x}-$ ray was performed to rule out pulmonary tuberculosis and MRI (Nuclear Magnetic Resonance) revealed a continuity solution in the posterior wall of the caecum with a collection that extended into the lateral wall of the pelvis. Affecting the iliac component of the right iliac psoas muscle, finding suggestive of a tumoral lesion, probably mucinous adenocarcinoma of the perforated caecum. Patient undergoes right hemicolectomy with histopathological report of blind tumor with macroscopic perforation present of type adenocarcinoma of high grade mucinous pattern with poor lymphoid response, extension to the serosa with perforation, metastasis to 2 lymph nodes and tumor implants to mesogastrium, classification pT4N1M1. Management was initiated with drainage, antibiotic therapy, iron, and the process of referral to the oncology clinic began. The case allowed us to recognize the operational performance of the diagnostic tests and the importance of their integration with the semiology of the patient, elements that were fundamental to reach an accurate diagnosis

Keywords: Psoas abscess, adenocarcinoma mucinous, suppuration

*Autor de correspondencia: Lina María Ortiz Sierra linama-ortiz@juanncorpas.edu.co Como citar: Ortiz Sierra, LM, Bernal Ferreira, G, Duarte, AM. Absceso de Psoas Secundario a un Adenocarcinoma Mucinoso de Colon Perforado: Semiología. Reporte de Caso. Revista Cuarzo 2019;25(1):21-27.

Recibido: 12 de febrero de 2019 Aceptado: 24 de mayo de 2019 Publicado: 30 de junio de 2019 DOI: https://doi.org/10.26752/cuarzo.v25.n1.425 


\section{INTRODUCCIÓN}

E 1 absceso de psoas, es una colección purulenta localizada en el compartimento de los músculos iliaco y psoas, que se encuentra en relación con órganos como: riñones, colon sigmoide, ciego, linfáticos, yeyuno, apéndice, páncreas, aorta abdominal y uréter (1). Este se puede clasificar en primario o secundario, dependiendo de la presencia o ausencia de una enfermedad subyacente. El absceso primario, ocurre por diseminación hematógena de una infección oculta sin demostración de foco infeccioso, el absceso de psoas secundario a su vez se presenta cuando el foco de infección es continuo o a distancia, la enfermedad de Crohn es una causa llamativa de presentación en estos casos. Los pacientes con intervención quirúrgica en estas zonas presentan también riesgo de desarrollar absceso de psoas secundario $(2,3)$.

Los abscesos de psoas primarios ocupan en África y Asia el $99 \%$ en Europa el $17 \%$ y América del Norte $61 \%$. Es más prevalente en el sexo masculino y se localiza con mayor frecuencia al lado derecho (4). Solo en un $20 \%$ de los casos se puede llegar al factor causal. La mayoría de los abscesos primarios son monomicrobiales (Staphylococcus spp), ocupando el $93 \%$ de los casos, mientras que el absceso de psoas secundario suele ser polymicrobial en el $27 \%$ de los casos, distribuyéndose de la siguiente manera: Staphylococcus aureus $(18 \%-90 \%)$ Escherichia coli $(9 \%-21 \%)$ Proteus mirabilis, Mycobacterium tuberculosis (36\%) Streptococcus grupo B (9\%) Bacteroides fragilis, Pseudomonas aeruginosa, Prevotella sp. Streptococcus mitis y Brucella spp. Salmonella enteritis (9\%) En la actualidad, se describe una distribución global para el absceso primario de psoas entre el $30-45 \%$ y para el secundario entre $55-70 \%$. El primario se presenta en menores de 30 años y se asocian con S. aureus entre el $85-90 \%$ (5).

El $80 \%$ de los abscesos secundarios se originan del tracto gastrointestinal, sin embargo, la literatura menciona que las causas más frecuentes de absceso de psoas secundario son las de tejido óseo, como lo son la osteomielitis vertebral en un 26,6 $\%$, seguida del absceso epidural, mientras que en el grupo de las gastrointestinales la más frecuente es la diverticulitis con un $5,5 \%$ y el cáncer colorrectal tan solo con un 1,8\%, afirma también que el absceso de psoas secundario a perforación de cáncer de colon es extremadamente raro y esta presentación atípica retrasa el diagnóstico de cáncer de colon generando una mayor morbimortalidad. En el $30 \%$ de los pacientes con absceso de psoas se presenta la triada clásica conformada por fiebre, dolor lumbar y limitación del movimiento de la cadera, derivada de su inervación por las raíces L2, L3 y L4. Otros síntomas pueden ser nauseas, pérdida de peso, anorexia, dolor lumbar $(91 \%)$ de los casos, la fiebre en el $(75 \%)$ y signo de psoas positivo ( $85 \%)(6)$.

El hemograma revela presencia de leucocitosis $>10.000 / \mathrm{ml}$ o $15.000 / \mathrm{ml}$ en el $83 \%$ de los casos, anemia, hemoglobina $<11$ $\mathrm{g} / \mathrm{dL}$ en un $42,6 \%$, la trombocitosis es menos frecuente se presencia en el $27 \%$ de los pacientes, mientras que la velocidad de sedimentación $>50$ se ve en el $73 \%$ de los casos.

El Gold estándar basados el rendimiento operativo de la prueba la sensibilidad de la RMN es de $88 \%$, mientras que, para la TAC simple, TAC mejorada reportan una sensibilidad para el absceso de psoas de $78 \%, 86 \%$ respectivamente. La RMN es útil cuando la patología es tumoral o existe una infección adyacente a los cuerpos o canales vertebrales, la ultrasonografía muestra la presencia de sombra o de gas en la zona, esta no suele ser definitiva, también se pueden ver lesiones hipodensas infiltración rodeada de grasa; la resonancia magnética es el examen más utilizado ya que permite una adecuada visualización de los tejidos blandos y estructuras adyacentes y una especial visualización de los cuerpos vertebrales. Un estudio realizado en el hospital de Kimitsu Chuo, China demostró que, a partir de los seis días posteriores al inicio de los síntomas, la sensibilidad de cada modalidad fue del $100 \%$. Mientras que la sensibilidad desde el primer día hasta los cinco días fue solo del $33 \%, 50 \%$ y $50 \%$, respectivamente este sugirió que ambas modalidades pueden pasar por alto un absceso en su etapa temprana (7).

\section{REPORTE DE CASO}

Paciente masculino de 63 años, residente en la ciudad de Girardot, albañil, sin ninguna morbimortalidad referida, manifiesta tener una vida tranquila Ingresa el 4 de marzo del 2018 a una institución de salud con cuadro clínico de 1 día de evolución, consistente en dolor en flanco y fosa iliaca derecha asociado a fiebre. Al examen de ingreso presenta taquicardia sin fiebre, no se evidencian signo de irritación peritoneal. Se ordenan paraclínicos donde encuentran: hemoglobina $5 \mathrm{mg} / \mathrm{dL}$ y VCM 62 acompañado de trombocitosis, endoscopia que mostró moniliasis esofágica, colonoscopia que reporta hemorroides e hipertrofia prostática benigna con regular preparación, tomografía axial computarizada (TAC) de abdomen evidencia colección en musculo iliaco derecho de $118 * 89 * 70 \mathrm{~mm}$ el cual es drenado, reporte del cultivo negativo y Ziehl Neelsen negativo con respuesta leucocitaria abundante por lo que se inició manejo intrahospitalario con lincosamina y penicilina por 28 días y es dado de alta.

El 4 de mayo del 2018 reingresa a una nueva institución prestadora de salud por reinicio de dolor en fosa iliaca derecha irradiado a miembro inferior derecho, sin fiebre con pérdida importante de peso. Se realizó nueva tomografía encontrando: absceso de psoas, el cual se drena por medio de catéter, deciden remitir a nuestra institución. A su ingreso se observa paciente con tensión arterial $110 / 70 \mathrm{mmHg}$ frecuencia cardiaca 79 latidos por minuto, frecuencia respiratoria de 16 respiraciones por minuto, pulso oximetría $93 \%$ al medio ambiente. Al examen físico se evidencia paciente pálido en regulares condiciones generales delgado con un peso $64 \mathrm{~kg}$ y un IMC 21.63, se observa en abdomen la presencia de catéter sobre cresta iliaca derecha con salida de escaso líquido seroso purulento. Durante su estadía en el hospital se toman paraclínicos (ver Tabla 1). 
Tabla 1: Paraclínicos Junio del 2018

\begin{tabular}{|c|c|c|}
\hline \multicolumn{3}{|c|}{ EXAMEN } \\
\hline & & Fecha: $6 / 06 / 2018$ \\
\hline \multicolumn{3}{|c|}{ Bioquímica: } \\
\hline- & BUN: $11.8 \mathrm{mg} / \mathrm{dl}$ & \\
\hline - & Sodio: $141 \mathrm{mEq} / 1$ & \\
\hline - & Potasio: $4.47 \mathrm{mEq} / 1$ & \\
\hline- & Cloro: $103 \mathrm{mEq} / 1$ & \\
\hline - & Calcio: $9 \mathrm{mEq} / \mathrm{l}$ & \\
\hline - & Proteína c reactiva: 5.88 & \\
\hline - & Hierro total 18 & \\
\hline- & Capacidad de combinación de hierro: 333.9 & \\
\hline - & Saturación de transferrina: 5.5 & \\
\hline - & Glucosa en suero u otro fluido: $91.5 \mathrm{mg} / \mathrm{dL}$ & \\
\hline- & Creatinina $: 0.73 \mathrm{mg} / \mathrm{dL}$ & \\
\hline \multicolumn{3}{|c|}{ Endocrinología: } \\
\hline- & Ferritina: 55,6 & \\
\hline \multicolumn{3}{|c|}{ Hematología: } \\
\hline- & Leucocitos: $6.85 \mathrm{~K} / \mathrm{uL}$ & \\
\hline - & Neutrófilos: $61.9 \%$ & \\
\hline- & Linfocitos: $21.9 \%$ & \\
\hline - & Monocitos: $7.4 \%$ & \\
\hline - & Eosinófilos: $8.5 \%$ & \\
\hline - & Basófilos: $0.30 \%$ & \\
\hline - & Recuento de eritrocitos: $5.02 \mathrm{M} / \mathrm{uL}$ & \\
\hline - & Hematocrito: $37.9 \%$ & \\
\hline - & Hemoglobina: $11.5 \mathrm{mg} / \mathrm{dL}$ & \\
\hline- & MCV: $75.5 \mathrm{fL}$ & \\
\hline- & MCH: 22.9 pg & \\
\hline - & MCHC: $30.3 \mathrm{~g} / \mathrm{dL}$ & \\
\hline - & RDW: $17.7 \%$ & \\
\hline - & Recuento de plaquetas automatizado: $818 \mathrm{~K} / \mathrm{uL}$ & \\
\hline - & MPV: 9.3 & \\
\hline - & PT: 15.9 & \\
\hline- & INR: 1.21 & \\
\hline- & TTP: 32.10 & \\
\hline \multicolumn{3}{|c|}{ Microbiología: } \\
\hline- & Coloración gram: negativo & \\
\hline- & Cultivo para microorganismos: negativo & \\
\hline
\end{tabular}

Fecha: 7/06/2018

Parcial de orina

Color: amarillo

Aspecto: limpio

Densidad: 1015

$\mathrm{pH}: 7$

leucos/esterasas: (-)

nitritos: (-)

proteínas: $(-)$

glucosa: normal

cetonas: (-)

urobilinógeno: normal

bilirrubina: (-)

eritrocitos: (-)

bacterias: escasas

células epiteliales: 1

leucos: 2

hematíes: $1 \mathrm{mg}$

Fecha: $13 / 06 / 2018$

\section{Endocrinología:}

Antígeno especifico de próstata: 0.65

Antígeno carcinoembrionario: 17.90

Alfa fetoproteina: 5.42

Antígeno de cáncer de tubo digestivo ca19- 9: 95.80

Fecha: $25 / 06 / 2018$

\footnotetext{
Hematología

Leucocitos: $7.45 \mathrm{~K} / \mathrm{uL}$

Neutrófilos: $54.20 \%$

Linfocitos: $22.8 \%$

Monocitos: $9.7 \%$

Eosinofilos: $12.5 \%$

Basófilos :0.80 \%

Recuento de eritrocitos: $4.47 \mathrm{~K} / \mathrm{uL}$

Hematocrito: $34.6 \%$
}

Hemoglobina: $10.5 \mathrm{mg} / \mathrm{dl}$

MCV: $77.4 \mathrm{fL}$

MCH: $23.5 \mathrm{pg}$

MCHC: $30.3 \mathrm{~g} / \mathrm{dL}$

RDW: $20.4 \%$

Recuento de plaquetas automatizado: $365 \mathrm{~K} / \mathrm{uL}$

MPV: 9.4

TP: 14.4

INR: 1.09

TTP: 24

Fecha: 26/06/2018

\section{Bioquímica:}

BUN: $17.9 \mathrm{mEq} / 1$

Sodio: $142 \mathrm{mEq} / 1$

Potasio: $4.47 \mathrm{mEq} / 1$

Cloro: $106.6 \mathrm{mEq} / 1$

Calcio: $8.6 \mathrm{mEq} / 1$

Creatinina $0.87 \mathrm{mg} / \mathrm{dL}$

Fecha: $28 / 06 / 2018$

\section{Hematología}

Leucocitos: $14.34 \mathrm{~K} / \mathrm{uL}$

Neutrófilos: $82.9 \%$

Linfocitos: $7.3 \%$

Monocitos: $6.9 \%$

Eosinófilos: $2.80 \%$

Basófilos: $0.10 \%$

Recuento de eritrocitos: $4.06 \mathrm{~K} / \mathrm{uL}$

Hematocrito: $32 \mathrm{mg} / \mathrm{dL}$

Hemoglobina: $9.8 \mathrm{mg} / \mathrm{dL}$

MCV: $78.8 \mathrm{fL}$

MCH: $24.1 \mathrm{pg}$

MCHC: $30.6 \mathrm{~g} / \mathrm{dL}$

RDW: $21 \%$

Recuento de plaquetas automatizado: $341 \mathrm{~K} / \mathrm{uL}$ MPV: 9.3

Fuente: Autores

La TAC y las características del dolor, el antecedente de drenajes a repetición y el tiempo de evolución del cuadro clínico hicieron pensar como posible diagnostico una espondilodiscitis por lo que se realizó una radiografía de tórax para descartar tuberculosis pulmonar y una ecografía de tejidos blandos la cual mostró imagen con ecogenicidad de 59×56*85 mm sobre el músculo del psoas, colección de $70 * 42 * 80$, la cual fue drenada por radiología intervencionista y RMN ya que es el Gold estándar para espondilodiscitis con un $96 \%$ de especificidad y un $92 \%$ de sensibilidad (8). Esta reveló solución de continuidad en la pared posterior del ciego, con colección que se extendía en la pared lateral de la pelvis, afectando el componente iliaco del músculo psoas iliaco derecho, la lesión era heterogénea con proyecciones papilares, con leves cambios inflamatorios y hallazgo sugestivo a lesión tumoral, probablemente adenocarcinoma mucinoso de ciego perforado con extensión en los tejidos blandos de la pared pélvica lateral. Con estos resultados se procedió a realizar una colonoscopia con biopsia la cual fue de inspección anal normal, tacto rectal esfínter normotónico, no masas, ampolla vacía, guante sin sangre, anoscopia se observan paquetes hemorroidales internos posterolaterales que superan la línea dentada se explora hasta colon derecho donde se observa lesión exofítica que compromete el $80 \%$ de la circunferencia, adenocarcinoma moderadamente diferenciado con focos mucoproductores en un 30\% infiltrante, sin invasión vascular ni perineural. 


\section{MANEJO}

Se inició tratamiento antibiótico empírico desde el ingreso a nuestra institución este se instauro durante los siguientes 10 días con ampicilina sulbactam 3 gramos intravenoso cada 6 horas, metronidazol 500 miligramos intravenoso cada 8 horas y albendazol tableta de $200 \mathrm{mg}$ al día durante cinco días. Se decidió llevar al paciente a un procedimiento quirúrgico, hemicolectomia derecha del ciego íleon terminal y colon derecho de $32 \mathrm{~cm}$, encontrando tumor en el ciego de $5.0 \times 5.0 \mathrm{x}$ $3.0 \mathrm{~cm}$ con perforación macroscópica presente de tipo adenocarcinoma mucinoso $80 \%$ de alto grado con respuesta linfoide intramural y peritumoral mínima extendido hasta la serosa con perforación, metástasis a 2 ganglios y con implantes tumorales al mesogastro, mucinosa que drena hacia peritoneo, siembras peritoneales diseminadas por todo epiplón menor (ligamento gastrohepatico, ligamento redondo epiplón mayor cara anterior del estómago y el meso intestinal desde su raíz) clasificación pT4N1M1 (ver imágenes).

Imagen 1: Compromiso Mural con Formación de Lagos de Mucina y Glándulas Neoplásicas.

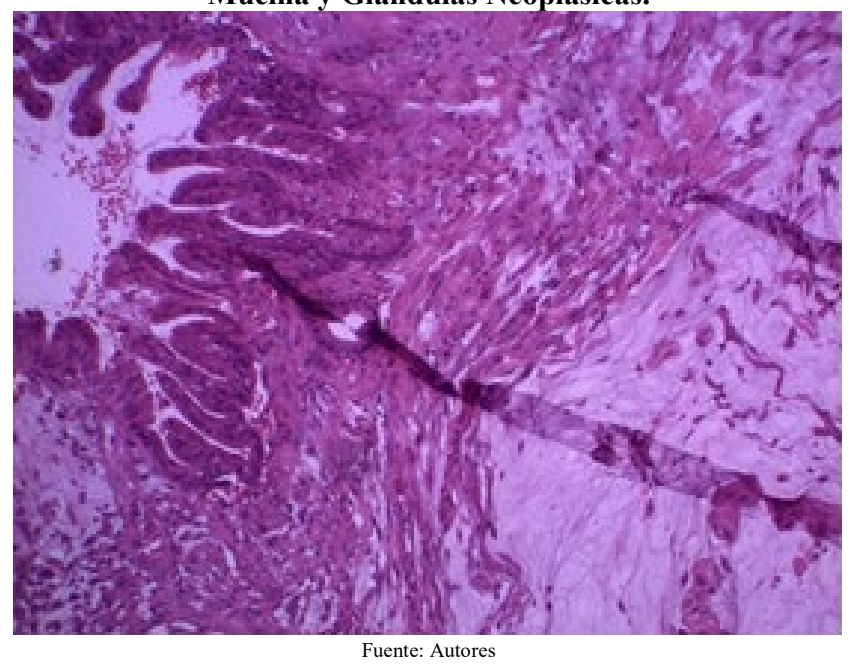

Imagen 2: Compromiso Mural con Formación de Lagos de Mucina y Glándulas Neoplásicas Disecando la Capa Muscular.

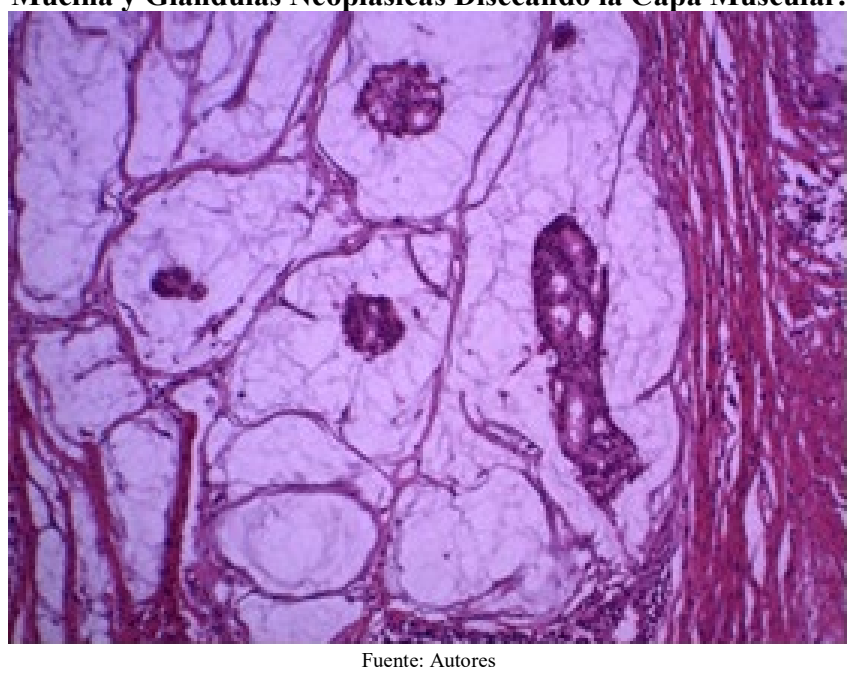

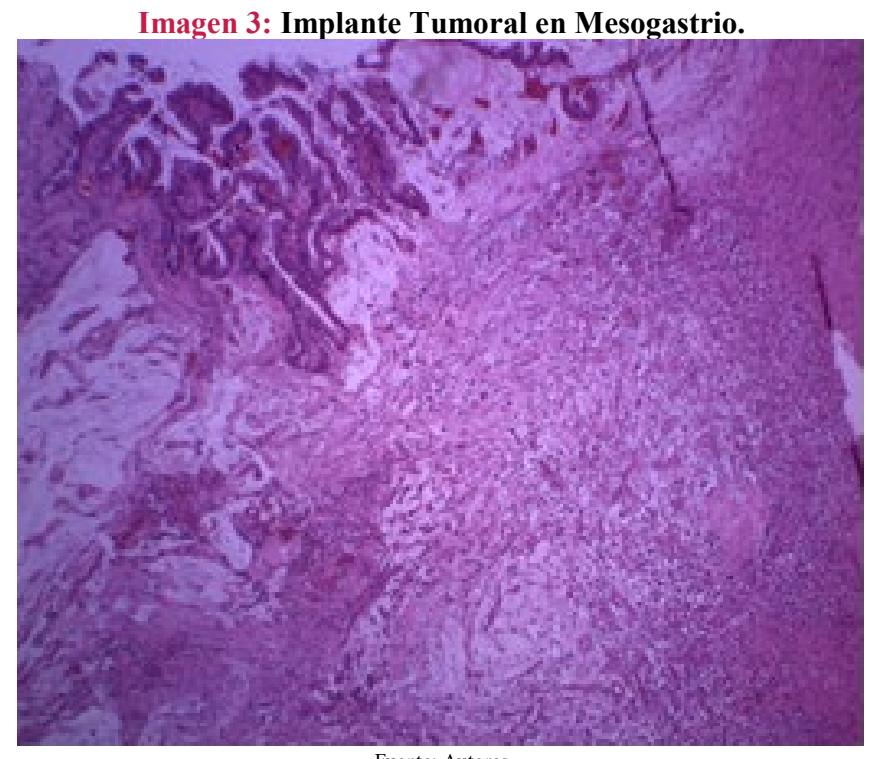

Fuente: Autores

Imagen 4: Compromiso Metastásico en Ganglio Linfático Regional.

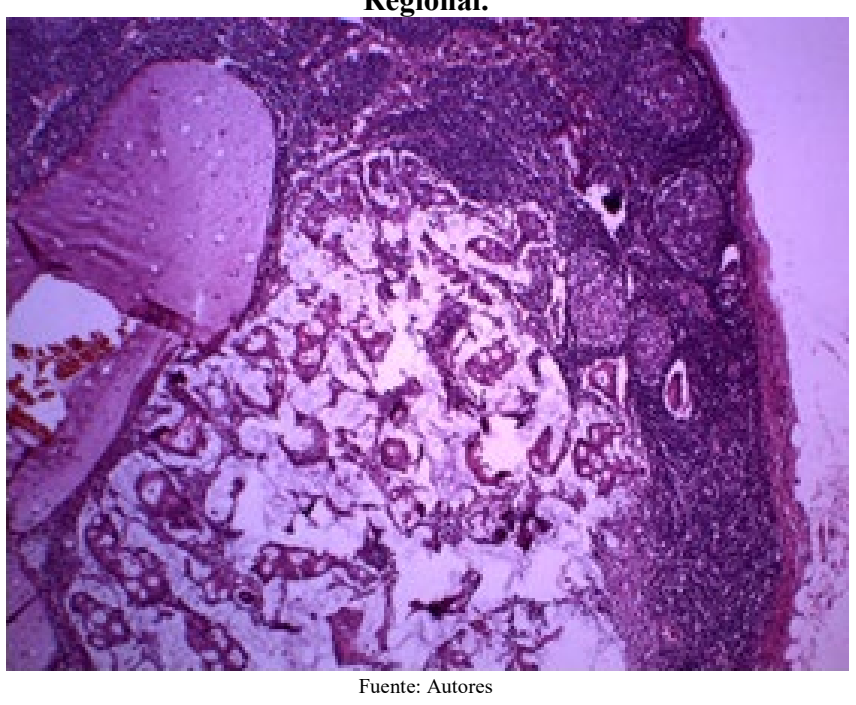

El paciente continuo en manejo con el servicio de cirugía allí se vigiló evolución clínica se le realizaron limpiezas a la herida una vez al día, cuantificación de drenaje con dren Jackson el cual fue retirado en el trascurso de 3 días, culminó antibiótico instaurado y tras posoperatorio favorable y no presentar eventos adversos se decidió dar egreso al paciente siendo interconsulta do por el servicio de oncología para continuar con su tratamiento.

Tabla 1: Evolución Clínica del Paciente. HALLAZGO CLÍNICO

Fecha: 04/03/2018

(Revisión de HC Extra Institucional)

Dolor en flanco derecho y fosa iliaca derecha irradiado a miembro inferior derecho, taquicardia, taquipnea, sugieren apendicitis. Fecha: 04/03/2018

(Revisión de HC Extra Institucional)

Toma de paraclínicos con evidencia de anemia normocítica heterogenia, trombocitosis y eosinofilia. Tomografía axial computarizada de abdomen 
con colección en músculo iliaco de 118 × 80 × 70 el cual drena radiología intervencionista.

\section{Fecha: 04/03/2018}

(Revisión de HC Extra Institucional)

Endoscopia reporta esofagitis grado I, colonoscopia con hemorragias e hiperplasia prostática benigna. Endoscopia reporta esofagitis grado I, colonoscopia con hemorragias e hiperplasia prostática benigna.

Fecha: 04/03/2018

(Revisión de HC Extra Institucional)

Tomografía axial computarizada de abdomen reporta colección heterogenia de $9 \times 56 \times 85$ con catéter de drenaje, y nueva colección heterogenia encima del musculo psoas de $70 \times 42 \times 80$.

Fecha: 04/03/2018

(Revisión de HC Extra Institucional)

Sospecha de posible inmunosupresión por esofagitis moniliasica. Fecha: 1/06/2018

Ingresa a la institución con absceso cutáneo en flanco derecho con dren. Fecha: 2/06/2018

Se diagnostica como absceso de Psoas. Fecha: 6/06/2018

Se solicita ecografía de tejidos blandos, resonancia magnética nuclear de abdomen para descartar posible espondilodiscitis o patologías retroperitoneales y radiografía de tórax para descartar tuberculosis pulmonar y se solicita valoración por nutrición. Fecha: 7/06/2018

Cultivo de secreción negativo.

Fecha: 8/06/2018

Radiografía de tórax normal, descartan tuberculosis pulmonar. Fecha: 12/06/2018

Cultivo de secreción negativo.

Fecha: 13/06/2018

Resonancia magnética nuclear sugestiva de lesión tumoral compatible con carcinoma mucinoso del ciego perforado con extensión en tejidos blandos de la pared pélvica lateral, por lo cual solicitan biomarcadores tumorales y colonoscopia.

Fecha: 15/06/2018

Colonoscopia reporta cáncer de colón derecho en espera biopsia. Fecha: 17/06/2018

Paciente con uso extenso de antibiótico por lo que se solicita valoración por epidemiologia y valoración por radiología intervencionista por drenaje escaso.

Fecha: 21/06/2018

Colonoscopia resultado inspección: hemorroides externas tacto rectal: normal anoscopia: hemorroides internas grado I.

colonoscopia: se explora hasta colon derecho donde se observa lesión exofítica que compromete el $80 \%$ de la circunferencia preparación: mala,

por la presencia de materia fecal liquida en todo el trayecto tiempo de salida: 6 minutos biopsia: lesión de colon derecho.

\section{Fecha: 25/06/2018}

Se realiza hemicolectomía derecha ileo transverso anastomosis y omentectomía parcial más biopsia de peritoneal.

hemicolectomía derecha con hallazgos de tumor de ciego con extensión más allá de la serosa, adherido a peritoneo parietal perforado con secreción

mucinosa que se drena hacia retroperitoneo. Siembras peritoneales

diseminadas por todo el epiplón menor (ligamento gastrohepático),

ligamento redondo, epiplón mayor, cara anterior del estómago y meso intestinal desde su raíz, siembras peritoneales a nivel del peritoneo parietal pélvico.

\section{Fecha: 27/06/2018}

Por persistencia del dolor se pide valoración con clínica del dolor los cuales manejan con analgésica por catéter peridural con bupivacaina y fentanilo.

fecha: $28 / 06 / 2018$

Retiro de catéter peridural.

Fecha: 1/07/2018

Se solicita estudio de triglicéridos del dren de Jackson para descartar colección asociada y tomografía axial computarizada de abdomen contrastada.

Fecha: $2 / 07 / 2018$

Reporte de tomografía con signos de íleo obstructivo con edema de paredes de asas intestinales delgada y gruesa que sugieren ileítis sin poder identificar opacificación del marco cólico. Imagen compatible con absceso

localizado en la musculatura anterior al iliaco derecho, presencia de catéteres de drenaje en fosa iliaca derecha.
Fecha: 3/07/2018

Paciente con reporte de triglicéridos normales, por lo que se descarta algún proceso infeccioso y se solicita ecografía para evaluar funcionalidad del dren y posteriormente retiro.

Fecha: 4/07/2018

Ecografía reporta colección en fosa iliaca derecha y disfunción de catéter, se solicita retirar dren por radiografía intervencionista.

Fecha: 5/07/2018

Radiografía intervencionista retira dren.

Fecha: 6/07/2018

Egreso con cita control por los servicios de oncología, cirugía y clínica del dolor, se dan signos de alarma, recomendaciones e incapacidad por 60 días.

Fuente: Autores

\section{DISCUSIÓN}

El absceso de psoas secundario es una identidad poco común lo que dificulta el diagnostico etiológico, se expuso el caso de un absceso de psoas secundario a perforación de cáncer de colon el cual es extremadamente raro, ocurre en un 1,8\% de la población, esta presentación atípica retrasa el diagnóstico de cáncer de colon generando una mayor morbimortalidad, la tomografía computarizada confirma el diagnostico en la mayoría de los pacientes $(71 \%)$, seguido por la resonancia magnética nuclear (26\%), este último es útil en pacientes en los que se sospecha una infección a estructuras adyacentes, los signos y síntomas suelen ser inespecíficos, representando un reto para su oportuno diagnóstico (8).

El diagnóstico de absceso se realiza cuando se presencia dolor, fiebre, masa palpable, leucocitosis y se pueden evidenciar pérdida de peso y anemia, esto también podría sugerir un tumor gastrointestinal como en el caso que se reportó (9). La RMN fue de gran ayuda, debido a que permitió identificar la lesión tumoral de adenocarcinoma mucinoso de ciego y su extensión. El cáncer colorrectal es el más común en la región de América, cada año se producen 240.000 casos nuevos y se registran aproximadamente 112.000 muertes, Canadá, Uruguay y Barbados presentan las tasas de incidencia más altas, mientras que en los países de América Central son más bajas. Sin embargo, la Organización Panamericana de la Salud y la Organización Mundial de la Salud estiman que para el 2030 la incidencia de cáncer aumentará en un $60 \%$ (10). Las neoplasias son la tercera causa de muerte en Colombia y el cáncer de colon se encuentra como la cuarta causa de muerte por neoplasias en hombres y la tercera causa en mujeres. Adenocarcinoma mucinoso rectal es un subtipo histológico que representa entre el $5 \%-15 \%$ del cáncer rectal, este se caracteriza por un acumulo abundante de mucina que excede el $50 \%$. El termino carcinoma mucinoso se designa a aquellos tumores en los que la secreción de moco contribuye al crecimiento tumoral, estos se pueden clasificar según el porcentaje de mucina $50 \%$ u 80 $\%$ como en el caso de nuestro paciente. Según la Organización Mundial de la Salud se requiere de un $50 \%$ para considerarse mucinoso $(11,12)$. Entre el 19 - $40 \%$ del adenocarcinoma no mucinoso se localiza en el colon derecho, mientras que la mayoría se encuentra en el colon izquierdo. Sin embargo, se ha demostrado que la prevalencia de adenocarcinoma mucinoso es igual en ambos segmentos (13). Estudios recientes han 
diferenciado lo clínico-patológico y el pronóstico de los pacientes con cáncer de colon derecho o izquierdo debido a su origen embrionario diferente, suplemento sanguíneo, inervación, drenaje linfático y ambiente del lumen generando síntomas diferentes en cada uno de los casos, tumores en el segmento derecho como en el caso expuesto presentan síntomas como anemia microcítica y pérdida de peso, mientras que tumores en el segmento izquierdo se manifiestan con cambios en los hábitos intestinales y hemorragia rectal; su pronóstico es malo debido a que es detectado cuando este ya se encuentra en estados muy avanzados con una gran proliferación metastásica, infiltración de serosa y diseminación peritoneal, se sabe que estos tienen una alta tasa de extensión; la razón de su comportamiento agresivo no es realmente clara, estudios demuestran que esto pude deberse a la excesiva producción de moco y disección de los planos tisulares del órgano comprometido. Adicional a esto, el fluido producido es absorbido por el sistema linfático favoreciendo de esta manera la propagación del tumor a los ganglios linfáticos regionales $(14,15)$.

\section{CONCLUSIONES}

Se presentó el caso de un paciente con absceso de psoas iliaco, secundario a un adenocarcinoma mucinoso perforado del ciego en estado avanzado, es importante resaltar todas las etiologías que lo pueden generar, independientemente de la poca frecuencia que esta tenga, como en el caso presentado. La semiología es de suma importancia para el diagnóstico, ya que como se ha venido mencionando, el cáncer de colon derecho se asocia a pérdida de peso progresiva y anemia, características que pudieron orientar a un diagnóstico más acertado. La RMN demuestra ser un examen de gran utilidad para la detección de cáncer de colon perforado hacia psoas. Es importante realizar un diagnóstico oportuno y un tratamiento cuyos objetivos sean reducir la mortalidad y la morbilidad ya que incide positivamente en el desarrollo y desenlace de la enfermedad.

Agradecimientos: Agradecer principalmente a la Fundación Universitaria Juan N. Corpas y a la Clínica Juan N. Corpas, quienes nos brindaron su apoyo y disposición para la realización de este proyecto. Al Doctor Víctor Hugo Forero, quien nos dio su aprobación y de esta manera logramos continuar con nuestro trabajo. A nuestro docente el Doctor Gonzalo Bernal, quien nos guió y asesoró durante el abordaje y desarrollo de este reporte de caso, agradecerle también por su acompañamiento y respaldo continuo. Al Doctor Andrés Mauricio Duarte, docente del área de patología de la Universidad quien nos ayudó con la recolección de las imágenes expuesta y nos guió en su interpretación y presentación de las mismas. Y agradecer a todas las personas que apoyaron y creyeron en el proyecto.

Conflicto de Interés: Los autores no declaran conflicto de interés.

\section{REFERENCIAS}

1. Askin A, Bayram KB, Demirdal US, Korkmaz MB, Gurgan AD, Inci MF. An Easily Overlooked Presentation of Malignant Psoas Abscess: Hip Pain [Internet]. Case Reports in Orthopedics. 2015 [citado 9 de noviembre de 2018]. Disponible en: https://www.hindawi.com/journals/crior/2015/410872/abs/

2. Tabrizian P, Nguyen SQ, Greenstein A, Rajhbeharrysingh U, Divino CM. Management and treatment of iliopsoas abscess. Arch Surg Chic Ill 1960. octubre de 2009;144(10):946-9. [citado 9 de noviembre de 2018]. Disponible en: https://www.ncbi.nlm.nih.gov/pubmed/19841363

3. I H Mallick, M H Thoufeeq, T P Rajendran. Iliopsoas abscesses. 10 Febr 2004 [Internet]. 1 de diciembre de 2003;80:459-462. Disponible en: https://www.ncbi.nlm.nih.gov/pmc/articles/PMC1743075/pdf/v0 80p00459.pdf

4. Ricci MA, Rose FB, Meyer KK. Pyogenic psoas abscess: worldwide variations in etiology. World J Surg. octubre de 1986;10(5):834-43. [citado 9 de noviembre de 2018]. Disponible en: https://www.ncbi.nlm.nih.gov/pubmed/3776220

5. JUAN FERNANDO MEDINA, MD*, VÍCTOR HUGO VIVAS, MD**. Absceso del psoas: revisión de la literatura y estado actual. 2004. 19(3):9. [citado 9 de noviembre de 2018]. Disponible

en: file:///C:/Users/Lina $\% 20 \mathrm{Mar} \% \mathrm{C} 3 \% \mathrm{ADa} /$ Downloads/absceso $\% 2$ 0del\%20psoas.pdf

6. Ouellette L, Hamati M, Flannigan M, Singh M, Bush C, Jones J. Epidemiology of and risk factors for iliopsoas abscess in a large community-based study. Am J Emerg Med [Internet]. 15 de mayo de 2018 [citado 9 de noviembre de 2018];0(0). Disponible en: https://www.ajemjournal.com/article/S0735-6757(18)30398$\underline{\mathrm{X} / \text { fulltext }}$

7. Toshihiko Takada, Kazuhiko Terada, Hideki Kajiwara and Yoshiyuki Ohira. Limitations of Using Imaging Diagnosis for Psoas Abscess in Its Early Stage Med [Internet]. 15 de mayo de 2018 [citado 9 de noviembre de 2018];0(0). Disponible en: file:///C:/Users/Lina $\% 20 \mathrm{Mar} \% \mathrm{C} 3 \% \mathrm{ADa} /$ Downloads/TAC $\% 20 \mathrm{E}$ N\%20Absceso\%20del\%20psoas.pdf

8. Herren C, Jung N, Pishnamaz M, Breuninger M, Siewe J, Sobottke R. Spondylodiscitis: Diagnosis and Treatment Options. Dtsch Arztebl Int. 2017;114(51-52):875-882. doi:10.3238/arztebl.2017.0875 [Internet]. 15 de mayo de 2018 [citado 9 de noviembre de 2018];0(0). Disponible en: https://www.ncbi.nlm.nih.gov/pmc/articles/PMC5769318/

9. Askin A, Bayram KB, Demirdal US, Korkmaz MB, Gurgan AD, Inci MF. An easily overlooked presentation of malignant psoas abscess: hip pain. Case Rep Orthop. 2015;2015:410872. [citado 9 de noviembre de 2018]. Disponible en: https://www.ncbi.nlm.nih.gov/pubmed/17471312

10.

11. Navarro López V, Ramos JM, Meseguer V, Pérez Arellano JL, Serrano R, García Ordóñez MA, et al. Microbiology and outcome of iliopsoas abscess in 124 patients. Medicine (Baltimore). marzo de 2009;88(2):120-30. [citado 9 de noviembre de 2018]. Disponible en: https://www.ncbi.nlm.nih.gov/pubmed/?term=Microbiology+an $\mathrm{d}+$ outcome + of + iliopsoas + abscess + in $+124+$ patients

12. Taiwo B. Psoas abscess: a primer for the internist. South Med J. enero de 2001;94(1):2-5. [citado 9 de noviembre de 2018];2014. Disponible en: file:///C:/Users/Lina\%20Mar\%C3\%ADa/Downloads/Psoas\%20 Abscess.pdf

13. Cayon A, https://www.facebook.com/pahowho. OPS/OMS | Cáncer colorrectal [Internet]. Pan American Health Organization / World Health Organization. 2016 [citado 9 de noviembre de 
2018].

Disponible

en:

https://www.paho.org/hq/index.php?option=com content\&view $=$ article\&id $=11761$ : colorectal-cancer\&Itemid=41765\&lang $=$ es

14. asis-nacional-2017.pdf [Internet]. [citado 9 de noviembre de 2018]. Disponible https://www.minsalud.gov.co/sites/rid/Lists/BibliotecaDigital/RI DE/VS/ED/PSP/asis-nacional-2017.pdf [citado 9 de noviembre de 2018]. Disponible

en: https://www.minsalud.gov.co/sites/rid/Lists/BibliotecaDigital/RI DE/VS/ED/PSP/asis-nacional-2017.pdf

15. SOCIALIZACIÓN ALCANCE OBJETIVOS GAI -Cancer Colon y Recto.pdf [Internet]. [citado 9 de noviembre de 2018]. Disponible en: https://www.minsalud.gov.co/Documentos\%20y\%20Publicacio nes/SOCIALIZACI\%C3\%93N\%20ALCANCE\%20OBJETIVO S\%20GAI\%20-Cancer\%20Colon\%20y\%20Recto.pdf 